\title{
An Efficient Route Discovery in Manets with Improved Route Lifetime
}

\author{
S. Priyadsrini, T. M. Navamani, and Venkatesh Mahadevan
}

\begin{abstract}
Mobile Ad hoc Networks consist of mobile nodes with limited battery power. The critical issue for routing in mobile ad hoc network is how to select a stable path with longer lifetime since mobility and power drain of a node causes frequent path failure. This path failure causes frequent route discovery which affects the performance of the routing protocol. The path failure also increases computational overhead of the nodes. Hence, in this paper, we propose three algorithms to estimate the stability of the route by considering the energy drain of the nodes and the rate of mobility. Finally we implement our proposed algorithms in AODV and the performance is evaluated against the original AODV. Our protocol improves the network performance and reduces the computation overhead by avoiding frequent route discovery since we select a stable path with longest life time. With the help of network simulator we substantiate that our proposed protocol performs better than the existing stability-based routing protocols with improved packet delivery ratio and reduced routing overhead.
\end{abstract}

Index Terms-Mobile ad hoc network, aodv protocol, route lifetime, routing failures, routing overhead, packet delivery ratio.

\section{INTRODUCTION}

Mobile Ad hoc Network (MANET) consists of many mobile nodes with wireless communication that can communicate with each other without any physical infrastructure. So it is called as infrastructure less network. Each node can perform the role of a host as well as a router. Hence the nodes which are out of transmission range can be accessed by routing through intermediate nodes. Often, hosts in a MANET operate with limited batteries and can roam freely towards any direction at any speed. The power exhaustion of some nodes and the mobility nature of nodes cause frequent topology changes. So the path between nodes or group of nodes may change periodically.

The node which wants to transmit data packets first needs to discover the route to the destination using route discovery process of different routing protocols. There are two kinds of routing protocols, one is reactive or ondemand routing protocol, and another is proactive or table-driven routing protocol. In mobile ad hoc networks, a host may exhaust its power or move away without giving any notice to its cooperative nodes, causing changes in network topology, and thus these changes

Manuscript received March 8, 2012; revised May 11, 2012.

S. Priyadsrini is with the Department of Computer Science and Engineering, Kingston Engineering College, Tamilnadu, (e-mail: priyait46@gmail.com)

T. M. Navamani is with Easwari Engineering College, TamilNadu, (email: navsmi2001@yahoo.com)

V. Mahadevan is from Swinburne University of Technology, Australia(e-mail: venkatsumee@hotmail.com) may significantly degrade the performance of the routing protocol. So the route needs to be discovered with longer route lifetime with fewer changes. As the route consists of number of wireless links, the route lifetime depends on the life time of nodes and individual links. The route discovery without considering the lifetime of the route leads to frequent failure and thereby to route discovery. As a result the computational overhead of the routing protocol increases considerably.

The remainder of the paper is organized as follows. Section II reviews the related works. Section III covers the steps involved in discovering the route with longest route

Lifetime. Implementation details and the simulation results present in the Section IV. Section V concludes this paper.

\section{RELATED WORKS}

In signal stability-based adaptive routing schemes (SSA) [1], each link is classified as a strong one or a weak one, depending on the received signal strength. A mobile node only processes RREQ that is received from a strong link. Functionally, the SSA protocol consists of two protocols, namely Forwarding Protocol (FP) and the Dynamic Routing Protocol (DRP). It is very sensitive to the particular configuration of the ad hoc network. Also it should consider the unpredictable mobility nature and the location instability of the nodes.

An analytical framework for the characterization of link behaviour in MANETs to accurately characterize the lifetime of a wireless link is presented in [2]. $\mathrm{Xi} \mathrm{Wu}$, et.al computed lifetime of a link through a two-state Markov model and that the analytical solution follows closely the results obtained through discrete event simulations. The advantage of this framework is that it accurately describes link dynamics as a function of node mobility. The link stability prediction-based routing (LSPR) algorithm [3] usesrelative motion and the distance between two neighbour nodes to evaluate the mean link duration to predict link stability. In LSPR algorithm, the mid nodes forward RREQs after delay which is decided by the mean link duration predicted. Furthermore a forwarding rule which can reduce the number of RREQs forwarded by preceding neighbour nodes is designed. But this protocol is not considering the lifetime of the node.

A stability-enhanced routing for mobile ad hoc networks was proposed in [4]. The previous stability-oriented routing algorithms usually focus on how to discover a stable route, but rarely consider the adaptability of constructed route to the change of node's motion. The link expiration time (LET), which is used to assess the stability of link, is calculated accurately in company with the discovery of some available 
stable routes in reactive manner. Based on the updated LET, the discovery of alternative stable route is determined, which can ensure the continuous transmission of data.

The energy drain rate of a node is affected not only by its own but also by its neighbouring data flows as well. The Network preserves connectivity by choosing a route according to the remaining battery life of nodes along the route. The path may be selected with minimum total transmission power when there exist multiple paths. It is necessary that all nodes through these paths have sufficient residual battery power and has the largest packet transmission capacity among multiple paths. In lifetimeprediction routing (LPR) algorithm [5], each node attempts to estimate its battery lifetime based on its residual energy and its past activity. The above algorithms used welldefined metrics to evaluate the lifetime of nodes. However, most of them ignored the mobility of mobile hosts, and thus, it seems that they are more suitable for static networks. Based on these observations we propose an efficient route discovery scheme that considers the mobility pattern and lifetime of nodes.

\section{ROUTE DISCOVERY WITH LONGEST LIFETIME}

The source needs to discover the route to the destination before transmitting any packets. This proposed protocol considers the lifetime of the route as a metric for route discovery process. The route consists of multiple links and the route is broken if any of the link fails. Thus the route lifetime becomes the minimum lifetime of all links in this route. Every link is formed by two adjacent mobile nodes, which have limited battery energy and can roam freely, and it is broken if any of the two nodes is not alive due to exhaustion of energy or if these two nodes move out of each other's transmission range. The Link Life Time (LLT) includes both the node lifetime and the connection lifetime. We introduce Connection Lifetime LCi to represent the estimated lifetime of the connection $\mathrm{Ci}$, and it only depends on their relative mobility and distance of nodes $\mathrm{Ni}-1$ and $\mathrm{Ni}$ at a given time. The term LNi denotes the estimated battery Lifetime of Node Ni. Then, the lifetime of the link Li is expressed as the minimum value of ( $\mathrm{LCi}, \mathrm{LNi}-1, \mathrm{LNi}$ ).

The lifetime of route $\mathrm{P}$ is expressed as the minimum

Value of the lifetime of nodes and connections involved in route P. Assume that $\sum$ represents the set of all nodes in Route $P$ and that $€$ is the set of all the connections in route $\mathrm{P}$. Thus, the lifetime $L p$ of route $\mathrm{P}$ can be expressed as

$$
\begin{gathered}
T p=\min \left(\mathrm{TN}_{i}, L C_{i}\right) \\
\mathrm{N}_{\mathrm{i}} \in \sum, \mathrm{Ci} \in €
\end{gathered}
$$

From (1), the lifetime $L p$ of route $\mathrm{P}$ is estimated from the lifetime of each node and each connection.

\section{A. Node Lifetime Prediction}

The Lifetime of the node is calculated based on its residual energy and its past history because the active node that is used for many data-transmissions consumed more energy and have shorter lifetime. The term $E_{i}$ represents the current residual energy of node $i$, and $e v_{i}$ is the rate of energy depletion. $E_{i}$ can be obtained from a battery management instrument, and evi is the statistical value that is obtained from recent history. We use an exponentially weighted moving average method to estimate the energy drain rate evi. Every $\mathrm{T}$ seconds node $i$ reads the instantaneous residual energy value and the corresponding estimated energy drain rate evi is obtained. We observe from (1) that the route lifetime is the minimum of the node lifetime and the connection lifetime.

\section{B. Connection Lifetime Prediction}

Since two nodes of a stable connection are within the communication range of each other, the connection lifetime may last longer, and they are not a bottleneck in the route to which they belong. It is easier to model the mobility of nodes in a short period during which unstable connections last. Note that previous works [6] and [7] are based on this assumption. The connection time TCi depends on the relative motion between $\mathrm{Ni}$ and $\mathrm{Ni}-1$, and the connection is said to be broken when two nodes $(\mathrm{Ni}-1, \mathrm{Ni})$ are moving out of each other's radio transmission range. We need to measure the distance between nodes $\mathrm{Ni}$ and $\mathrm{Ni}-1$, and the relative velocity of these two mobile nodes. Easy method is to measure the received signal strength. Assuming that senders transmit packets with the same power level, a receiver can measure the received signal power strength when receiving a packet and then calculates the distance by directly applying the radio propagation model [8]. If the received signal power strength is lower than a threshold value, we regard this link as an unstable state and then calculate the connection time.

\section{Route Discovery}

Our proposed algorithms are implemented in AODV protocol as it is one of the popular on demand routing protocols and it is the combined protocol of DSDV and DSR. But this routing protocol was not considering the route lifetime and QoS parameters during route discovery. In our modified AODV protocol, the source which wants to discover the route to a particular destination forwards RREQ packet. The intermediate nodes calculate node lifetime and connection lifetime using the algorithms as described in the above section and it forwards the route request along with the computed information to next node till it reaches the destination. After RREQ reaches the destination, it forwards RREP to the source. Every intermediate node exchanges two control packets. So each node calculates the connection lifetime after it received the corresponding RREP. The Route Lifetime is calculated as the minimum of node and link lifetime and it is forwarded along with RREP to the source through the intermediate nodes. The source selects the path with the longest route lifetime

\section{IMPLEMENTATION}

\section{A. Simulation}

For our simulations, we use a discrete event-driven simulator NS version 2.34. Table I summarizes the more detailed simulation parameters.

The proposed system is simulated with the simulation 
time of 200s. The Tcl for proposed system has configured with wireless scenario. The AODV routing protocol is used, since it is a popular reactive routing protocol. Moreover AODV does not consider QOS and link stability in its route discovery process. Also the control overhead of route discovery is more compared to DSR [9]. Thus frequent path breaks lead to frequent route discovery which affects the network efficiency and also the overall performance of the routing protocol. So the route lifetime prediction algorithm is implemented in AODV to avoid these problems of AODV. There are 50 mobile nodes in the scenario. There are three source and three destinations. The node1, node 4 and node41 are the sources. The node9, node 15 and node 27 are the corresponding destinations. Each source forwards route request packet to find the route to the corresponding destination via the intermediate nodes at different times.

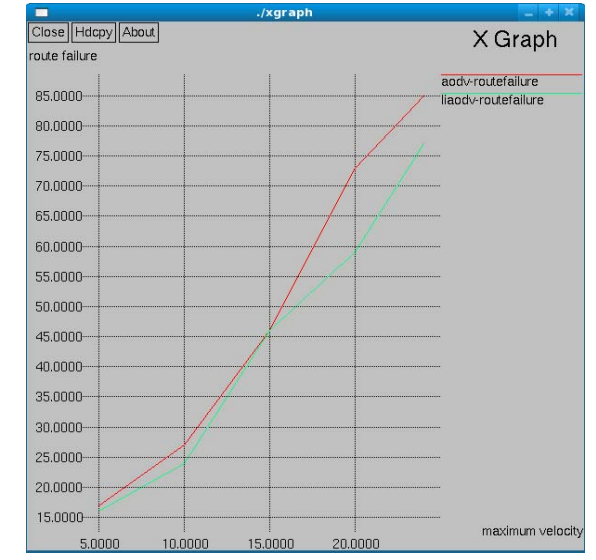

Fig. 1. Route failure comparison

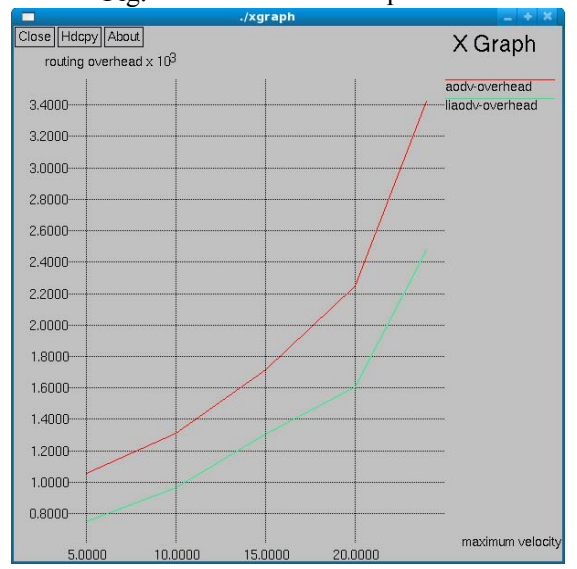

Fig. 2. Routing overhead comparison

\section{B. Simulation Results}

To evaluate the performance of the proposed system, we compared the performance of the enhanced AODV with route lifetime prediction algorithms with traditional AODV in terms of network packet delivery ratio, routing failures, and control packet overhead. The original AODV tends to find the shortest path from the source node to the destination node, ignoring the route lifetime. The proposed route lifetime prediction algorithm implemented in AODV performs better than the original AODV protocol in varying node velocity environments. Fig. 1 shows the advantage of the proposed protocol in terms of the number of routing failures. The routing failure occurs because of the node failure or the link failure. The proposed algorithm selects the path with longest route lifetime. As the route with lowest lifetime is eliminated and only the route with longest lifetime is selected for data forwarding, the number of link breakage is reduced.

TABLE I : SIMULATION PARAMETERS

\begin{tabular}{|c|c|}
\hline \multicolumn{1}{|c|}{ TABLE I : SIMULATION PARAMETERS } \\
\begin{tabular}{|c|c|} 
Simulation \\
time
\end{tabular} & 200s \\
\hline $\begin{array}{l}\text { Number of } \\
\text { Nodes }\end{array}$ & 50 \\
\hline MAC Type & MAC 802.11 \\
\hline $\begin{array}{c}\text { pr } \\
\text { Radio }\end{array}$ & Two Ray Ground \\
\hline $\begin{array}{l}\text { Energy } \\
\text { model }\end{array}$ & Energy model \\
\hline $\begin{array}{l}\text { Traffic Type } \\
\text { Routing } \\
\text { protocol }\end{array}$ & CBR \\
\hline $\begin{array}{l}\text { C } \\
\text { B }\end{array}$ & AODV \\
\hline \begin{tabular}{l}
$\mathrm{n}$ \\
\hline
\end{tabular} & Omni Antenna \\
\hline
\end{tabular}

Routing overhead is defined as the amount of routing control packets, including RREQ and RREP. In our proposed algorithm the number of route discovery is reduced as we are reducing the route failures. So the frequent route discovery is avoided which in turn reduces the node's computational and routing overhead involved in route discovery process. Fig. 2 shows the routing overhead of AODV and enhanced AODV with our proposed algorithm. The proposed protocol yields a significant improvement with the help of our proposed route lifetime-prediction algorithm, and its overhead is reduced as compared with AODV

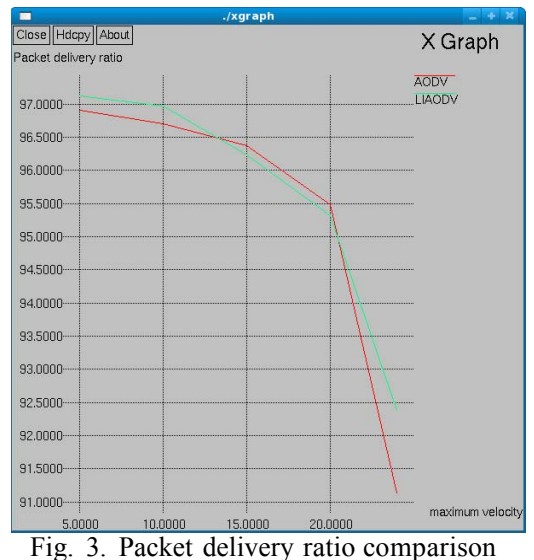

\section{CONCLUSION}

The algorithms to predict node lifetime, link lifetime, route lifetime are implemented in NS2.34 using AODV routing protocol. As our proposed route discovery process considers the lifetime of the route as the metric while selecting the route, the routing failure is minimized. This reduces the number of route discovery process and also the computation overhead of every node involved in route discovery process which affects the overall performance of routing protocol. To evaluate 
the performance, proposed protocol is compared with existing AODV with different node velocities. This shows that our proposed protocol reduces routing failure and route overhead. It also improves packet delivery ratio. Our future work includes the security for the proposed algorithm to discover the route with longer lifetime and without any malicious nodes which affects the performance

\section{REFERENCES}

[1] R. Dube, C. D. Rais, K. Y. Wang, and S. K. Tipathi, "Signal stability based adaptive routing (SSA) for ad hoc mobile networks," IEEE Papers Commun, vol. 4, no. 1, pp. 598-598, 2010.

[2] X. Wu, H. R. Sadjadpour, and J. J. Garcia-Luna-Aceves, "An analytical framework for the Characterization of link dynamics in MANETs," in Proc. IEEE Mil. Commun. Conf, 2006.

[3] X. Hu, J. Wang, and C. Wang, "Routing in Mobile AdHoc Networks," IEEE conference.
[4] X. Wu, J. Wang, and C. Wang, "Stability-enhanced routing for Mobile ad hoc Networks," presented at International Conference on Computer Design and Appliations (ICCDA 2010), 2010.

[5] M. Maleki, K. Dantu, and M. Pedram, "Lifetime prediction Routing in mobile ad hoc networks," in Proc. IEEE, 2008

[6] W. Su, S. J. Lee, and M. Gerla, "Mobility prediction and routing in ad hoc wireless networks," Int. J. Netw. Manage. IEEE transaction vol. 11, no. 1, pp. 3-30, 2011.

[7] P. Samar and S. B. Wicker, "On the behaviour of Communication links of A node in a multi-hop mobile environment," in Proc. Int. Symp. Mobile Ad Hoc Netw. Compute, pp. 145-156, 2004.

[8] T. K. Sarkar, Z. Ji, K. Kim, A. Medouri, and M. Salazar-Palma, “A survey of various propagation models for mobile communication," IEEE Antennas Propag. Mag, vol. 45, no. 3, pp. 51-82, June 2003.

[9] A. K. Yupta, H. Sadawarti, and A. K. Verma, "Performance analysis of AODV, DSR \& TORA routing protocols," IACSIT International Journal of Engineering and Technology, vol.2, no.2, pp.226-231, 2010. 